\title{
Imaging of SN 1993J
}

\author{
A. Alberdi ${ }^{1}$ and J.M. Marcaide ${ }^{2}$
}

${ }^{1}$ IAA-CSIC, Apdo. Correos 3004, 18080 Granada, Spain antxon@iaa.es

${ }^{2}$ Departamento de Astronomía, Universidad de Valencia, 46100 Burjassot, Spain

Summary. SN 1993J has been imaged with VLBI, and its angular expansion monitored, for almost ten years. The images show shell-like radio structures with almost circular symmetry. SN 1993J expands according to models of shock excited emission. The angular expansion has a changing deceleration rate and is best modeled with two different slopes. The swept-up mass estimate at an age of 3159 days $(\sim 0.4$ $\mathrm{M}_{\odot}$ ), comparable to the low-mass envelope, favors a binary scenario. The observed spectral index of SN 1993J has slowly flattened since age 1000 days onward ( $\alpha$ has changed from -1 to -0.67 at an age of 2820 days).

\section{Introduction}

SN 1993J is the radio supernova whose evolution has been monitored in greatest detail. It exploded on March 28th, 1993 and its radio emission was discovered a few days later (April 2 [22]; April 5 [15]). Its brightness and proximity has permitted unprecedented VLBI studies since the explosion.

It was first detected with VLBI on 25 April 1993. The observations were performed at $8.4 \mathrm{GHz}$ on the baseline between the Madrid DSN antenna DSS63 and Westford, with a fringe spacing of 1.4 milliarcseconds $([8,9]$; on the second IAU Circular, the position of the supernova was established with a precision better than 10 mas).

\subsection{Early Radio Size Estimates of SN 1993J}

Marcaide et al. [10] provided estimates for the angular size of SN 1993J 29 and 36 days after explosion (0.28 0.06 mas on May 1, 1993), using an optically thick uniform disk model to analyze their data.

Bartel et al. [1] made a series of VLBI observations at 8.4 and $22 \mathrm{GHz}$, from one to three months after the supernova explosion, and found that the supernova was circularly symmetric and its expansion showed no signs of deceleration. Using uniform disk models, they determined an expansion velocity of $2.98 \pm 0.08 \mu \mathrm{as} /$ day, which they found consistent with the maximum optical line velocities $(18,000 \pm 1,000 \mathrm{~km} / \mathrm{s})$ [21], thus indicating that the radio emission arose from the shock front resulting from the interaction between the SN-ejecta and the circumstellar medium. 


\subsection{Discovery of the Shell-like Radio Structure}

Marcaide et al. [11], at a supernova age of 239 days, showed that SN 1993J had a shell-like radio structure, the youngest ever discovered in a supernova. As seen in the Plate 1 (Fig. a), the radio shell was almost circularly symmetric, suggesting for the supernova explosion and the expanding radio shell a nearly spherical symmetry. The brightness distribution around the shell was quite uniform with enhanced emission in the south eastern part of the ring. Our estimate for the radio shell radius for 22 November 1993 (age 239 days) was $581 \pm 35 \mu$ as and yielded, assuming a zero-size at explosion, an average expansion rate for the shell radius of $2.43 \pm 0.15 \mu \mathrm{as} /$ day, lower than the value previously estimated by Bartel et al. [1]. We determined a ratio of shell thickness to outer radius of $0.3 \pm 0.1$.

The discovery of the shell structure was in agreement with the "Standard Interaction Model" (SIM) [5, 6]. In this model, the radio emission is assumed to be produced in a region of circumstellar gas (density profile $\rho_{c s m} \propto r^{-s}$; $\mathrm{s}=2$ corresponds to a steady wind) shocked by the outgoing ejecta (density profile $\rho_{e j} \propto r^{-n}$ ), thus giving rise to a "mini-shell" emission. For $\mathrm{n}>5$, selfsimilar solutions are possible: the radii of the discontinuity surface, forward shock and reverse shock are related and all evolve in time with the power law $\mathrm{R} \propto t^{m}$, where $\mathrm{t}$ is the time after explosion and the deceleration parameter, $m$, is given by $(n-3) /(n-s)$.

\section{Expansion of SN 1993J: An Exploding Star in Motion}

After the discovery of the shell-like radio structure, we initiated a VLBI observing program to monitor the supernova expansion in a manner free from modeling uncertainties. Our first results [12] provided the first movie of the angular expansion of a radio supernova. The movie included observations from day 182 to day 541 (see Plate 1, Fig. b). SN 1993J expanded with circular symmetry and a self-similar evolution: at the radio frequency of $8.4 \mathrm{GHz}$, the structure relative to the outer radius remained time invariant even though the outer radius was expanding. Assuming a free expansion, we found an average angular expansion rate of $2.39 \pm 0.03 \mu$ as/day. We also found that the region of enhanced emission at the south-eastern part of the ring persisted with time and that the region had the steepest spectral index $\left(\alpha \leq-1.5, S \propto \nu^{\alpha}\right)$.

Further observations at $5 \mathrm{GHz}$ over 1304 days showed that the expansion was decelerating $(\mathrm{m}=0.86 \pm 0.02)$ [13]. Rupen et al. [19] found a similar result $(\mathrm{m}=0.837 \pm 0.025)$. All images had a circular shape, and the azimuthal changes in the brightness distribution could be attributed to inhomogeneities in the CSM (clumping in the wind), irregularities in the ejecta or changes in the magnetic field. After 1304 days, the velocity of the shock front had decreased to $9000 \mathrm{~km} / \mathrm{sec}$. 


\section{Deceleration in the Expansion of SN 1993J}

Two research groups, one led by N. Bartel and the other by J.M. Marcaide, have monitored the angular expansion of SN 1993J for almost ten years [2, $3,4,12,13]$. The following conclusions can be drawn from their efforts:

\subsection{Evolution of the Radio Shell}

Along these years, the supernova has expanded rather isotropically (at least as seen in a $2 \mathrm{D}$ sky projection) showing a circular shell-like structure (deviations from circularity of the outer radius are smaller than $3 \%$ ). However, the brightness distribution has been systematically changing both with time and azimuth around the source center. We show in the Plate 1 (Fig. c) two images of SN 1993J obtained on 22 September 1999 (age 2369 days) and 7 November 2002 (age 3511 days). For comparison, both images are shown convolved with a circular beam of 2 mas. In the first map, there is enhanced emission at the south-eastern part of the ring and a gap at the north-western part, whereas in the second the brightness distribution has become more complex with hot spots formed within the shell, even at position angles where the emission was very low in the first map (i.e. at the south-western part of the shell). Part of these changes are due to artifacts of the image reconstruction process, but part of them might be real. However, one should emphasize that, despite those enhancements and hot spots, the shell emission is rather uniform.

One common characteristic for all epochs (published, and those shown in the Plate still unpublished), is that the emission in the central part of the shell is much lower than that corresponding to a spherical optically thin shell with uniform emissivity. This indicates the existence of an important absorption in the central part of the source $[4,14]$, likely due to intervening ionized ejecta [7].

\subsection{Determination of the Deceleration}

In their published work, the group led by Marcaide has based their determinations on measurements of the supernova size on the image plane. In this case, the source size inferred from a map depends on how the map is produced. Because of the finite beam size, a positive bias is introduced in the size estimate: the size measured is larger than the true size. The fractional bias would systematically decrease for a source increasing in size if the same beam were used for all observations. This would cause an spurious contribution to the deceleration. Such undesirable contribution can be largely avoided by using a "dynamical beam," that is, using each time a beam of size proportional to the actual source size [13]. Considering all the observing epochs at 3.6 and $6 \mathrm{~cm}$ (more than 25 observing epochs ranging from 72 to 3511 days), and fitting a single parameter to the data, Marcaide et al. have obtained 
a deceleration parameter of $\mathrm{m}=0.87 \pm 0.01$ (Supernova Radius $\mathrm{R} \propto t^{m}$; using a convolving beam $\theta_{b} \propto t^{1.0}$ ) and $\mathrm{m}=0.83 \pm 0.02$ (using a convolving beam $\left.\theta_{b} \propto t^{0.8}\right)$.

Adding the results for $30 \leq \mathrm{t} \leq 175$ days as given by Bartel et al. [2] to our data set, and using a dynamical beam that goes as $\theta_{b} \propto t^{0.8}$, we have reanalyzed our data through day 3511 allowing for a change of deceleration rate in the expansion of SN1993J (implying two deceleration parameters), that is, two straight lines and a time break in a log-log plot. We obtain the best fit with the following parameters: $m_{1}=0.93 \pm 0.06, m_{2}=0.82 \pm 0.01$, and $t_{\mathrm{br}}=383 \pm 121$ days (see Fig. 1). This fit has a reduced $\chi_{\nu}^{2}=2.18$. Analyzing the same data set with only one deceleration parameter we obtain $m=$ $0.83 \pm 0.01$, with a reduced $\chi_{\nu}^{2}=2.76$. Though the latter result is compatible with our earlier estimate [13], it has a slightly larger reduced $\chi_{\nu}^{2}$ than the fit with two deceleration regimes. It should be noted though that including the data from [2] affects very much the solution and that their data and ours have been obtained in different ways. See also [14] for a completely new approach. Their conclusions differ from those presented in this review.

The change in the angular growth rate of SN 1993J, if true, could be due to: i) a change in the density profile of the CSM caused by a changing mass loss rate of the progenitor and/or ii) a change in the density profile of the supernova ejecta (since $m=(n-3) /(n-s)$ in the SIM). Combining the deceleration rate determinations with the time dependence of the opacity due to an external medium $\left(\tau \propto t^{\delta}\right.$, with $\left.\delta=-(2 s-1) m, \delta=-1.99_{-0.16}^{+0.38}[20]\right)$ and assuming that $\delta$ has not changed with time, we found that for early epochs in the expansion ( $\mathrm{t} \leq 400$ days) $\mathrm{s}=1.52$ (density profile for the presupernova wind) and $n=22.7$ (ejecta density profile), while for later epochs $s=1.71$ and $\mathrm{n}=8.9$. This result indicates that the density profile of the CSM has changed with time, thus suggesting that the fast circumstellar wind was probably not steady.

However, there is increasing evidence that, besides free-free absorption, synchrotron self-absorption (SSA) is also relevant for SN 1993J [7, 16, 17]. In fact, Fransson and Björnsson (1998) [7] can explain the light curves assuming a standard $s=2$ density profile for the presupernova wind. In this case, the expansion deceleration could be associated with $n$ (ejecta density profile) changing from 14.9 to 7.6 from early to late times. The supernova emission is becoming progressively more dominated by swept-up material, with the evolution of the supernova governed by the interaction of the high energy particles in the shell with the surrounding medium.

Assuming $\mathrm{s}=2$ and typical values for the mass-loss rate and wind velocity $\left(\dot{M}=5 \times 10^{-5} \mathrm{M}_{\odot} \mathrm{yr}^{-1}, v_{w}=10 \mathrm{~km} / \mathrm{s}\right)$, the swept-up mass after 3157 days, $\sim 0.4 \mathrm{M}_{\odot}$, is comparable to the low-mass envelope, thus favoring a binary scenario.

Bartel et al. [2, 4] have also imaged SN 1993J and measured the supernova size. Their results are based on model fitting of the two-dimensional 
beam proportional to $t^{0.8}$

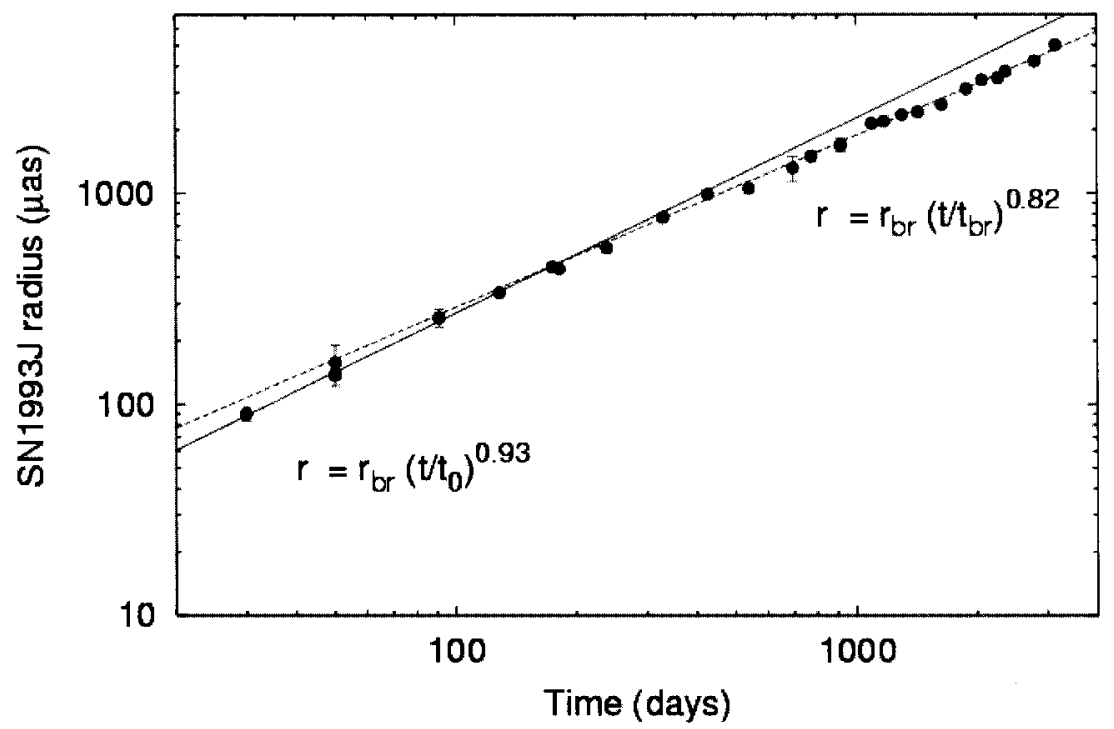

Fig. 1. Weighted least squares fit to the outer shell radius of SN1993J as a function of time since explosion, allowing for a change in its deceleration rate. The VLBI data up to $t \leq t_{\mathrm{br}}=403$ days (where the solid and dashed lines in the figure cross each other) can be well fitted by a power-law with index $m_{1}=0.93 \pm 0.06$ (solid line), while for $t \geq t_{\mathrm{br}}$ the best fit is given by a power-law of index $m_{2}=0.82 \pm 0.01$ (dashed line). Note that the scale is logarithmic. Our data and data from Bartel et al. [2] have been combined. The Bartel et al. data greatly affect the fit in the early days. See also the text for details about the dynamical beam used.

projection of a three-dimensional spherical shell of uniform emissivity to the calibrated uv-data set. They have also determined the supernova expansion, and have even claimed the detection of decreasing deceleration from age 1900 days onward. They find three different slopes: $m=0.919 \pm 0.019$ (age $30-300$ days), $\mathrm{m}=0.781 \pm 0.009$ (age $582-1893$ days) and $\mathrm{m}=0.860 \pm 0.011$ (age 1893-3164 days). According to them, these changes indicate that the evolution of SN 1993J is not self-similar, and are simultaneous with structural changes, line velocity changes and changes in the light curve decline. This time-dependent deceleration was predicted by Mioduszewski et al. [16], who calculated the time evolution of the expansion parameter from hydrodynamical simulations using an specific explosion model. In their interpretation, the interaction of features in the ejecta density structure with the reverse shock is relevant for the angular expansion. 


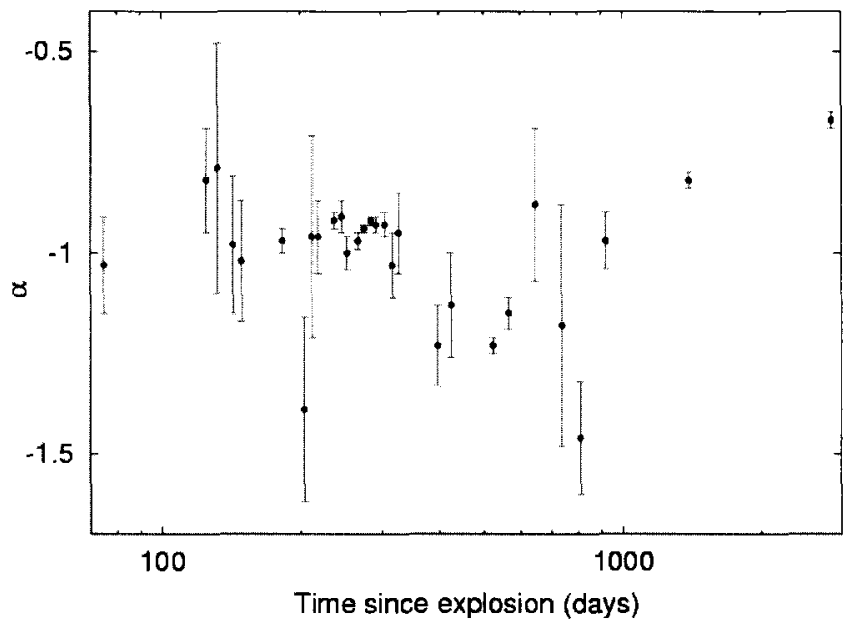

Fig. 2. Observed spectral index, $\alpha$, of SN1993J from $t \sim 70$ days up to $t=2820$ days, as obtained from fitting at each epoch the available VLA data to a synchrotron spectrum, partially suppressed by free-free absorption. After $t \sim 1000$ days, the spectrum is flattening. The data used for the fits come from K. Weiler (private communication), except for the last data point which is from [18].

\subsection{Determination of the Shell Width}

The thickness of the shell has been found to be $30 \pm 10 \%$ [11] (based on fits on the images) and $25 \pm 3 \%$ [4] (based on fits in the uv plane, taking into account the central absorption) of its outer radius. See [14] in this proceedings volume for a detailed account on this matter.

\section{Spectral Evolution of SN 1993J}

We have fitted the available radio continuum data of SN 1993J for the period from $\sim 70$ up to 2820 days after the explosion [18]. For all epochs, the spectra follow a power-law within the errors. The fit shows that the observed spectral index of SN 1993J has been slowly evolving since $t \sim 1000$ days onward, with $\alpha$ flattening from a value close to -1 to a value of $-0.67 \pm 0.02$ on day 2820 (see Fig. 2). A similar spectral evolution has also been reported by Bartel et al. [3].

The spectral evolution might suggest that radiative losses (mainly synchrotron), adiabatic cooling and ionization losses at the lowest frequencies contribute significantly to the integrated electron spectrum. Preliminary results from our group confirm the ongoing SN 1993J spectrum evolution, becoming progressively less steep (at least, up to age 3500 days where $\alpha \sim-0.55)$. 
For a power-law relativistic electron distribution, the spectral index of the electron distribution $(\gamma)$ is related to the observed spectral index by $\gamma=1-2 \alpha$. Our value of $\alpha$ on day 2820, translates into $\gamma=2.34 \pm 0.04$, which agrees well with the injected electron spectrum predicted by [7], $\gamma=2.1$, consistent with diffusive shock acceleration. These authors propose that a constant fraction of the shocked thermal electrons, characterized by a constant spectral index $\alpha=-0.55$, are injected and accelerated. At early stages, synchrotron losses dominate the cooling of the electrons, steepening the integrated electron spectrum up to a value of $\sim-1$. For epochs $t \geq 1000$ days, radiative losses become less relevant and the observed spectral index flattens progressively, getting closer to the spectral index associated with the injected electron population, which seems consistent with the observational results.

Fransson and Björnsson [7] have modeled the SN 1993J light curves assuming a standard $r^{-2}$ circumstellar medium and considering both free-free absorption and synchrotron self-absorption (SSA) (the importance of SSA was also discussed by other authors $[17,16])$. They determine a magnetic field of $\mathrm{B} \sim 64\left(R_{s} / 10^{15} \mathrm{~cm}\right)^{-1} \mathrm{G}$, which argues strongly for a turbulent amplification behind the shock. Fransson and Björnsson also determine that the number density of relativistic electrons scales as a fixed fraction of the thermal energy density behind the shock.

\section{Summary}

SN 1993J is to date the radio supernova whose evolution has been monitored in greatest detail and the one which holds best promise for a comprehensive theoretical-observational analysis. SN 1993J has been imaged, and its angular expansion monitored, for almost 10 years. The results obtained by the two groups carrying out VLBI observations of SN1993J show a general agreement and can be summed up and summarized as follows:

- The shell-like radio structure of SN 1993J has expanded according to models of shock excited emission, showing almost circular symmetry, thus suggesting that the shock front is expanding almost isotropically. The brightness distribution changes with azimuth around the source center and with time. No protrusions have been detected.

- The angular expansion may not be self-similar. The expansion may be best modeled with two slopes.

- The swept-up mass estimate $\left(0.4 \mathrm{M}_{\odot}\right.$ at 3157 days), comparable to the low-mass envelope, favors the binary scenario.

- A fit to the radio spectra of SN 1993J from $\sim 70$ up to 2820 days shows a clear, albeit slow, evolution with $\alpha$ changing from $\simeq-1$ to $\simeq-0.67$.

- Free-free absorption from the CSM and Synchrotron Self-Absorption are relevant in SN 1993J. 


\section{References}

1. N. Bartel et al. : Nature 368, 610 (1994)

2. N. Bartel et al. : Science 287, 112 (2000)

3. N. Bartel et al. : Astrophys. J. 581, 404 (2002)

4. M.F. Bietenholz, N. Bartel, M.P. Rupen: Astrophys. J. 368, 610 (2003)

5. R.A. Chevalier: Astrophys. J. 258, 790 (1982)

6. R.A. Chevalier: Astrophys. J. 259, 302 (1982)

7. C. Fransson, C.I. Björnsson: Astrophys. J. 509, 861 (1998)

8. J.M. Marcaide et al. : IAUC 5785 (1993)

9. J.M. Marcaide et al. : IAUC 5820 (1993)

10. J.M. Marcaide et al. : Astrophys. J. Lett. 424, L25 (1994)

11. J.M. Marcaide et al. : Nature 373, 44 (1995)

12. J.M. Marcaide et al. : Science 270, 1475 (1995)

13. J.M. Marcaide et al. : Astrophys. J. Lett. 486, L31 (1997)

14. J.M. Marcaide et al. : In: This proceedings

15. G.G. Pooley, D.A. Green: IAUC 5751 (1993)

16. A.J. Mioduszewski, V.V. Dwarkadas, L. Ball: Astrophys. J. 562, 869 (2001)

17. M.A. Pérez-Torres, A. Alberdi, J.M. Marcaide: Astron. Astrophys. 374, $997(2001)$

18. M.A. Pérez-Torres, A. Alberdi, J.M. Marcaide: Astron. Astrophys. 394, $71(2002)$

19. M.P. Rupen et al. : In: Radio Emission from Galactic and Extragalactic Compact Sources - IAU Coll. 64, ed. by J.A. Zensus, G.B. Taylor, J.M. Wrobel (ASP Conf. Series, Vol. 144, 1998) pp. 353-354

20. S.D. Van Dyk et al. : Astrophys. J. Lett. 432, L115 (1994)

21. J.C. Wheeler et al. : Astrophys. J. Lett. 417, L71 (1993)

22. K.W. Weiler, R.A. Sramek, S.D. van Dyk, N. Panagia: IAUC 5752 (1993) 

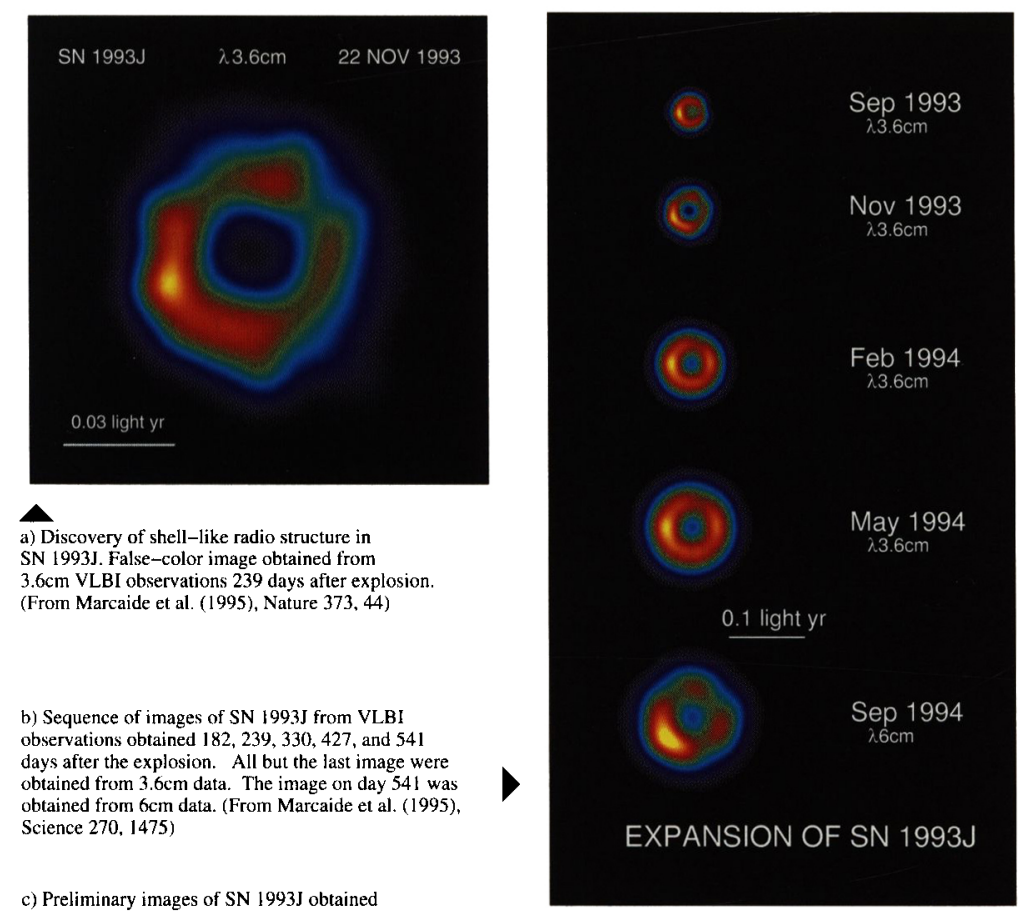

a) Discovery of shell-like radio structure in SN 1993J. False-color image obtained from $3.6 \mathrm{~cm}$ VLBI observations 239 days after explosion. (From Marcaide et al. (1995), Nature 373, 44)

b) Sequence of images of $\mathrm{SN} 1993 \mathrm{~J}$ from VLBI observations obtained $182,239,330,427$, and 541 days after the explosion. All but the last image were obtained from $3.6 \mathrm{~cm}$ data. The image on day 541 was obtained from $6 \mathrm{~cm}$ data. (From Marcaide et al. (1995), Science 270,1475

c) Preliminary images of SN $1993 \mathrm{~J}$ obtained from $6 \mathrm{~cm}$ VLBI observations 2396 days (left) and 3511 days (right) after the explosion.

$\checkmark$

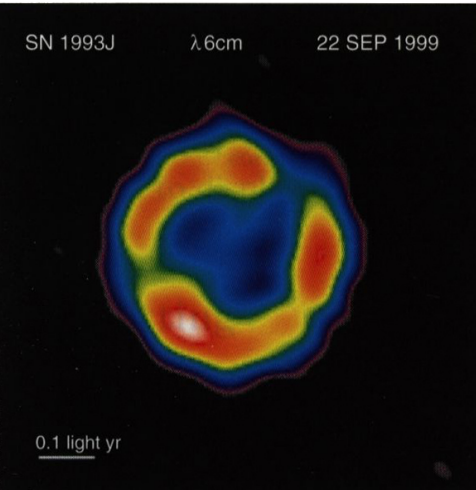

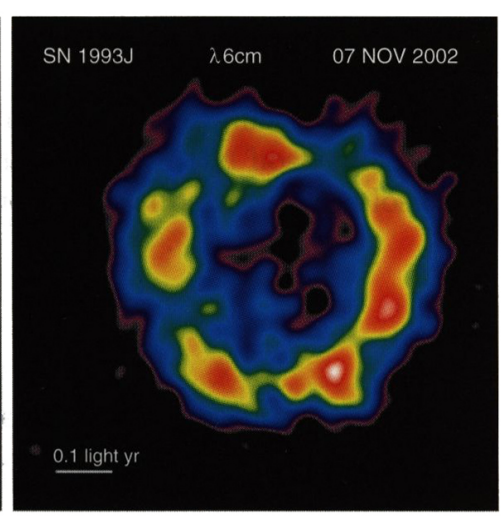

Plate (Alberdi \& Marcaide)

Plate 1. 